\title{
Wavefront outer scale and seeing measurements at San Pedro Mártir Observatory
}

\author{
R. Conan ${ }^{1}$, R. Avila ${ }^{2}$, L. J. Sánchez ${ }^{3}$, A. Ziad ${ }^{4}$, F. Martin ${ }^{4}$, J. Borgnino ${ }^{4}$, O. Harris ${ }^{5}$, S. I. González ${ }^{3}$, \\ R. Michel ${ }^{5}$, and D. Hiriart \\ 1 European Southern Observatory, Karl-Schwarzschild-Str. 2, 85748 Garching bei München, Germany \\ 2 Instituto de Astronomía UNAM, Apdo. Postal 72-3 (Xangari), 58089 Morelia Mich., México \\ e-mail: r.avila@astrosmo.unam.mx \\ 3 Instituto de Astronomía UNAM, Apdo. Postal 70-264, 04510 México D.F., México \\ e-mail: leonardo@astroscu. unam.mx \\ ${ }^{4}$ U.M.R. 6525 Astrophysique, Université de Nice Sophia Antipolis/CNRS, Parc Valrose, 06108 Nice Cedex 2, France \\ e-mail: Aziz.ZIAD@unice.fr, Francois.MARTIN@unice.fr, Julien.BORGNINO@unice.fr \\ 5 Instituto de Astronomía UNAM, Apdo. Postal 877, 22800 Ensenada B. C., México \\ e-mail: harris@astrosen.unam.mx, rmm@astrosen.unam.mx, hiriart@astrosen.unam.mx
}

Received 4 December 2001 / Accepted 10 September 2002

\begin{abstract}
The first measurements of the spatial coherence outer scale at the Observatorio Astronómico Nacional at San Pedro Mártir (OAN-SPM) are reported along with long term seeing measurements. These parameters were measured with the Generalized Seeing Monitor and with a Differential Image Motion Monitor. An instrumented mast was also used to measure the structure constant of the refractive index $C_{n}^{2}$ in the first $15 \mathrm{~m}$. Log-normal statistics were found for the seeing and for the outer scale, with median values of $0.92^{\prime \prime}$ and $27.0 \mathrm{~m}$, respectively. The distribution of the outer scale values is similar to that found in other observatories around the world, suggesting that the presence of trees in the OAN-SPM do not affect the outer scale values. Correlation studies suggest that large values of the seeing and the outer scale are likely to occur when the wind blows from the SSW. Further studies are recommended to confirm this tendency.
\end{abstract}

Key words. atmospheric effects - turbulence - site testing - techniques: high angular resolution instrumentation: adaptive optics

\section{Introduction}

In recent years, observational methods with High Angular Resolution in optical astronomy have seen a fast improvement and deployment, mainly of the leading techniques which are adaptive optics and long baseline interferometry. Both techniques benefit from a better modeling and knowledge of the optical effects of atmospheric turbulence. Important constraints on the design of instruments of these kinds are imposed by the models of optical atmospheric turbulence. These models need to be fed with on-site measurements in order to be as much realistic as possible. These on-site measurements are more easily provided with dedicated instruments as those used in this work, than with common astronomical instruments.

The main purpose here is to report measurements of the wavefront outer scale $\mathcal{L}_{0}$, of the isoplanatic angle $\theta_{0}$ and a study of the seeing at the Observatorio Astronómico Nacional at San Pedro Mártir (OAN-SPM), Baja California, México, held by the Instituto de Astronomía of the Universidad Nacional

Send offprint requests to: R. Conan, e-mail: rconan@eso.org
Autónoma de México (IA-UNAM). The measurements of $\mathcal{L}_{0}$ were obtained with the Generalized Seeing Monitor (GSM) from the "Département d'Astrophysique de l'Université de Nice Sophia-Antipolis" (DAUNSA). This instrument has been employed for similar studies at several observatories around the world: ESO La Silla (Chile), ESO Paranal (Chile), Oukaïmeden (Morocco), Maidanak (Uzbekistan) and Cerro Pachón (Chile). A Differential Image Motion Monitor (DIMM) was also used to measure the open-air seeing during different epochs. We used micro-thermal probes mounted on a dedicated 15-m-high mast to measure the optical turbulence strength in the first $15 \mathrm{~m}$. These measurements are used here only to be able to compare seeing measurements performed at different altitudes: The GSM and the DIMM were installed with a difference in altitude of $8 \mathrm{~m}$ in December 2000, and the DIMM was installed either at ground level or at 8-m hight in different epochs (see Sects. 3.3.1 and 3.3.2). A detailed study of the influence of the ground layer in the seeing at the OAN-SPM will be presented in a dedicated forthcoming paper. Finally an ultrasonic anemometer was used to measure the wind speed at an altitude of about $12 \mathrm{~m}$. 
The wavefront outer scale has never been measured before at the OAN-SPM. This parameter plays an important role for the adaptive optics programs under way at the IA-UNAM, and for the design of future new technology telescopes at the site. Moreover, an important question was whether the presence of trees in the Observatory area has an implication on the outer scale values. All the measurements reported here took place at the precise selected site for the telescope in project Telescopio Infrarrojo-óptico Mexicano (TIM). In Sect. 2, we describe the instruments used and the observations. The results are presented and discussed in Sect. 3.

\subsection{The Observatory site}

San Pedro Mártir (SPM) site of the Mexican Observatorio Astronómico Nacional is situated on the northern part of the Baja California peninsula. A complete description of the site can be found in previous papers (Avila et al. 1998; Tapia 1992; Hiriart et al. 1997; Alvarez 1969; Echevarría et al. 1998; Michel et al. 2001). Concerning optical turbulence studies, Avila et al. (1998) monitored the vertical distribution of the optical turbulence strength $C_{n}^{2}(z)$, using the Generalized Scidar of the DAUNSA, finding that the seeing originated in the first kilometer, in the free atmosphere, and in the whole atmosphere had median values of $0.56^{\prime \prime}, 0.45^{\prime \prime}$ and $0.78^{\prime \prime}$, respectively. These values were found when the Generalized Scidar was installed at the $2.1 \mathrm{~m}$ telescope and include dome seeing. Echevarría et al. (1998) reported a median open air seeing of 0.61 arcsec, obtained during an extensive (3-years) seeing campaign, using non-differential seeing monitors. Masciadri et al. (2002) presented evidence of a finite horizontal extension of turbulence layers at the site.

\section{Methods of measurements}

\subsection{Observations}

The observations performed with the GSM took place from 2000 December 3rd to 13th. The DIMM was in operation during that run, and additional data from 2000 May and October, and 2001 October are reported here (see Sect. 2.3). The instrumented mast delivered reliable data during 2000 May and 2000 December 6th to 9 th.

All atmospheric-turbulence parameters given here are calculated for a wavelength of $\lambda=0.5 \mu \mathrm{m}$ and for observations at zenith. The seeing angle $\epsilon$ corresponds to the full width at half maximum of a long-exposure image of a point source in a large telescope. The wavefront outer scale $\mathcal{L}_{0}$ corresponds to an average of the outer scale in the different turbulence layers $L_{0}(h)$, weighted by the vertical distribution of the optical-turbulence strength $C_{n}^{2}(h)$ Borgnino (1990). The isoplanatic angle $\theta_{0}$ is that relevant for adaptive optics imaging (Fried 1982).

\subsection{The Generalized Seeing Monitor}

This instrument was developed at the DAUNSA, France. Since its early development until its last missions, several articles provide extensive descriptions of the GSM and the interested

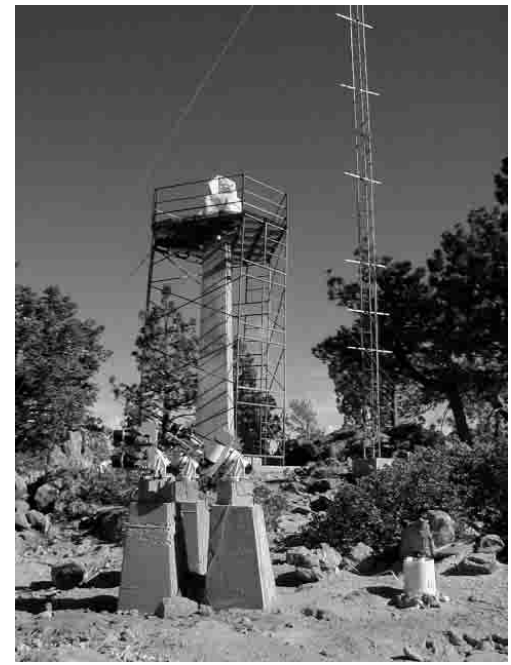

Fig. 1. Layout of the GSM (foreground), of the DIMM tower (leftbackground) and the mast (right-background).

readers could refer to these papers. The initial version of GSM is presented by Martin et al. (1994). Martin et al. (1998b) and Tokovinin et al. (1998b) present the current version of the instrument used for the measurements reported here. A detailed description and an analysis of the performances of the instrument is given by Ziad et al. (2000). We also refer the interested reader to the reports on the previous GSM runs (Tokovinin et al. 1998b; Martin et al. 1998b; Ehgamberdiev et al. 2000; Martin et al. 1998a).

At the OAN-SPM, the GSM modules and their mounts were installed on top of 3 concrete pillars, the telescopes being $1.5 \mathrm{~m}$ above ground. The layout of all the instruments is shown in Fig. 2. A wind-protective enclosure consisting of 2-m-high-nets was installed, covering South, East and West sides around the instrument. The angle of arrival (AA) were measured for two integration times: $5 \mathrm{~ms}$ and $10 \mathrm{~ms}$ and extrapolated to an integration time of $0 \mathrm{~ms}$ (Ziad et al. 2000). The AA are recorded during two minutes and processed to provide the Fried's parameter $r_{0}$, the wavefront outer scale $\mathcal{L}_{0}$ and the isoplanatic angle $\theta_{0}$. In the rest of the paper, we will convert $r_{0}$ into the seeing angle $\epsilon=0.10 \mathrm{~m} / r_{0}$ arcsec at $0.5 \mu \mathrm{m}$, which is the full width at half maximum of the point spread function of a seeing-limited telescope for an infinite outer scale.

\subsection{The Differential Image Motion Monitor}

A DIMM (Sarazin \& Roddier 1990) is a fairly well known instrument used to measure the seeing. We give here only a brief description. A complete presentation can be found in Vernin \& Muñoz-Tuñón (1995). The DIMM used in these observations was purchased at the company LHESA Electronique and was originally developed in a collaboration between the DAUNSA and the Instituto de Astrofísica de Canarias (Spain). It consists of a $20-\mathrm{cm}$ Celestron telescope supported by an equatorial mount. The entrance pupil of the telescope has a diaphragm that creates two 6-cm circular sub-pupils separated by $14 \mathrm{~cm}$. One of the sub-pupils has an optical wedge, so that on the focal plane two images of the observed point source are formed. 

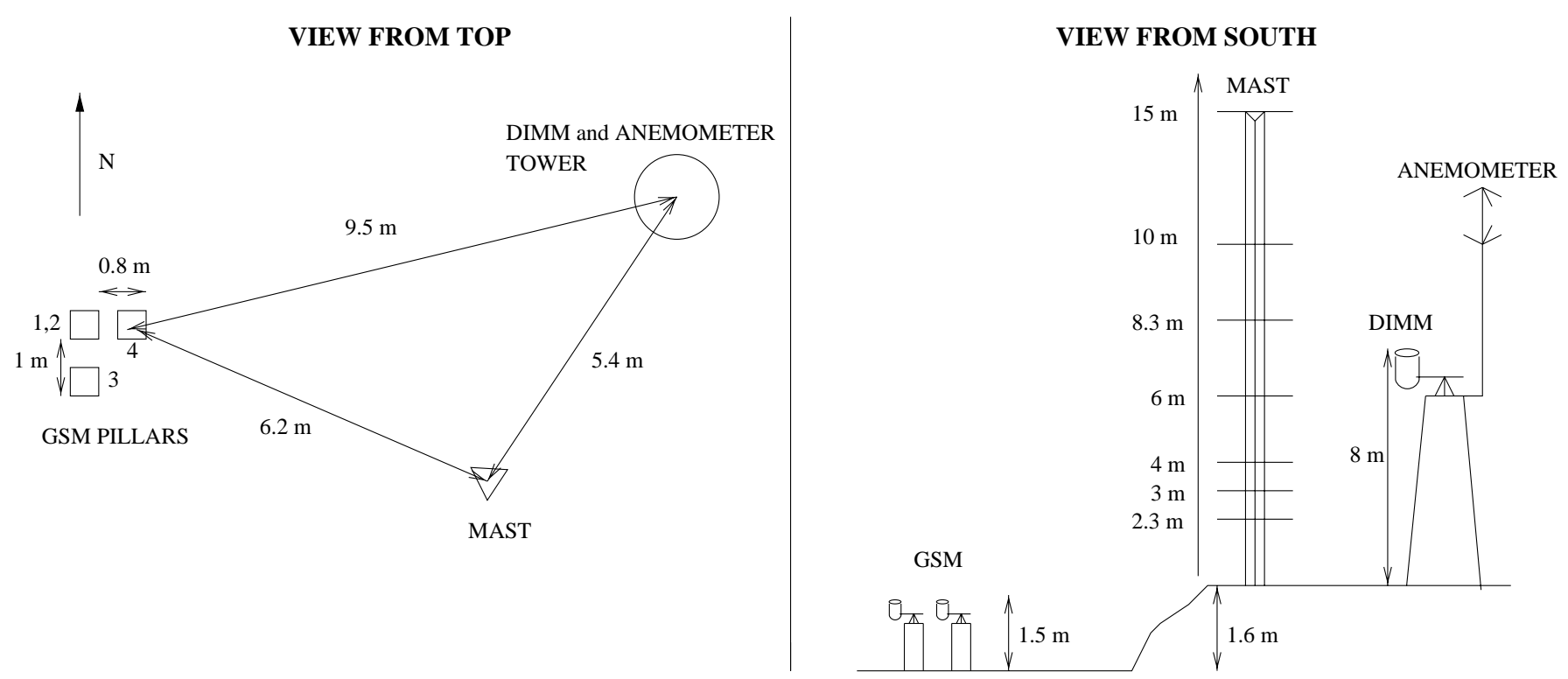

Fig. 2. Layout of the location of the instruments during the December 2000 campaign.

An intensified CCD records a focal plane frame every $20 \mathrm{~ms}$, with an exposure time of $10 \mathrm{~ms}$. Using a PC, the photo-center of each of the two star-images is determined for each frame. These photo-centers vary randomly as a consequence of atmospheric turbulence. From a set of 400 frames, the variance of the differential image positions is calculated and related to the seeing using the standard theory of optical turbulence (Roddier 1981). A seeing value corrected for the airmass factor is delivered every $30 \mathrm{~s}$ with an accuracy better than 0.1 arcsec.

We analyzed DIMM data obtained in different epochs: 7-22 May, 9 and 29 October, 1-14 December 2000 and 6-11 October 2001. During May and October 2000, the DIMM was installed on a low-altitude platform, that brought the entrance pupil $2 \mathrm{~m}$ above the ground, whereas in the December 2000 and October 2001 campaigns, the DIMM was installed on a tower, so that the pupil was $8 \mathrm{~m}$ above the ground. Only in December 2000 the other instruments were operating. The position of the DIMM relative to the other instruments during that campaign is shown in Fig. 2.

\section{Data analysis and discussion}

\subsection{GSM data summary}

The data summary for the GSM run (2000 December) is presented in Table 1. In the first column, we give the date and the remaining columns contain the nightly median values of the main atmospheric parameters measured by GSM, namely seeing $\epsilon_{\mathrm{GSM}}$, outer scale $\mathcal{L}_{0}$ and isoplanatic angle $\theta_{0}$. The bottom line contains the median values calculated from the whole data set of the campaign. A graphical representation of the ensemble of the GSM data is given in Fig. 3.
Table 1. GSM data summary.

\begin{tabular}{cccc}
\hline \hline Date & $\begin{array}{c}\epsilon_{\mathrm{GSM}} \\
\prime\end{array}$ & $\begin{array}{c}\mathcal{L}_{0} \\
\mathrm{~m}\end{array}$ & $\begin{array}{c}\theta_{0} \\
{ }^{\prime}\end{array}$ \\
\hline 03.12 & 0.97 & 30.1 & 2.29 \\
06.12 & 1.00 & 27.2 & 1.46 \\
07.12 & 1.21 & 49.2 & 2.16 \\
08.12 & 0.92 & 19.8 & 2.58 \\
10.12 & 1.19 & 25.0 & 1.62 \\
11.12 & 2.15 & 24.5 & 0.99 \\
12.12 & 2.35 & 36.1 & 2.92 \\
13.12 & 0.94 & 15.0 & 1.38 \\
\hline Median from & & & \\
whole data set & 1.15 & 27.0 & 2.05 \\
\hline
\end{tabular}

\subsection{The wavefront outer scale}

The median value of $\mathcal{L}_{0}$ measured in the whole observing campaign is equal to $27.0 \mathrm{~m}$. The histogram of $\mathcal{L}_{0}$, given in Fig. 4, shows a log-normal statistics.

An example of typical variations of $\mathcal{L}_{0}$ during a night is given in Fig. 5. The median value for this night is $20 \mathrm{~m}$ with a standard deviation of $16 \mathrm{~m}$. At the beginning of the night, a sudden high $\mathcal{L}_{0}$ value has been measured. This kind of large isolated values have been already noticed in the previous campaigns of the GSM and called "bursts".

All previous monitoring of $\mathcal{L}_{0}$, in different astronomical sites, gave also log-normal distributions for this parameter. The median values obtained at the different sites are very similar to each other, ranging from 24 to $31 \mathrm{~m}$ and in every case the dispersion of $\log \left(\mathcal{L}_{0}\right)$ is about 0.23 . 


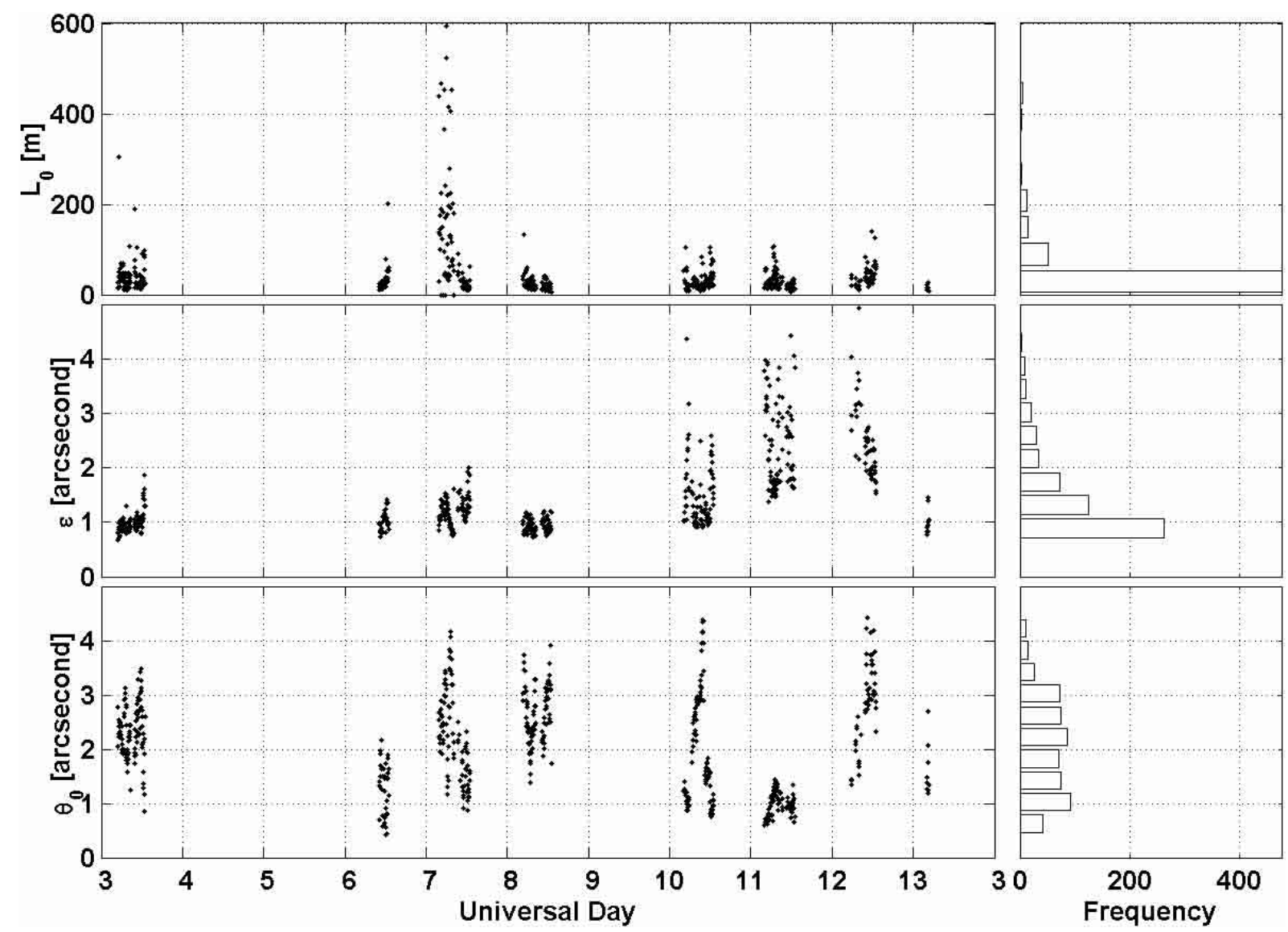

Fig. 3. Summary of the GSM data obtained at the OAN-SPM during the 8 nights of the run: the panels on the left show the evolution of the wavefront outer scale $\mathcal{L}_{0}$, seeing $\epsilon$ and isoplanatic angle $\theta_{0}$, while the panels on the right show the histograms of these parameters.

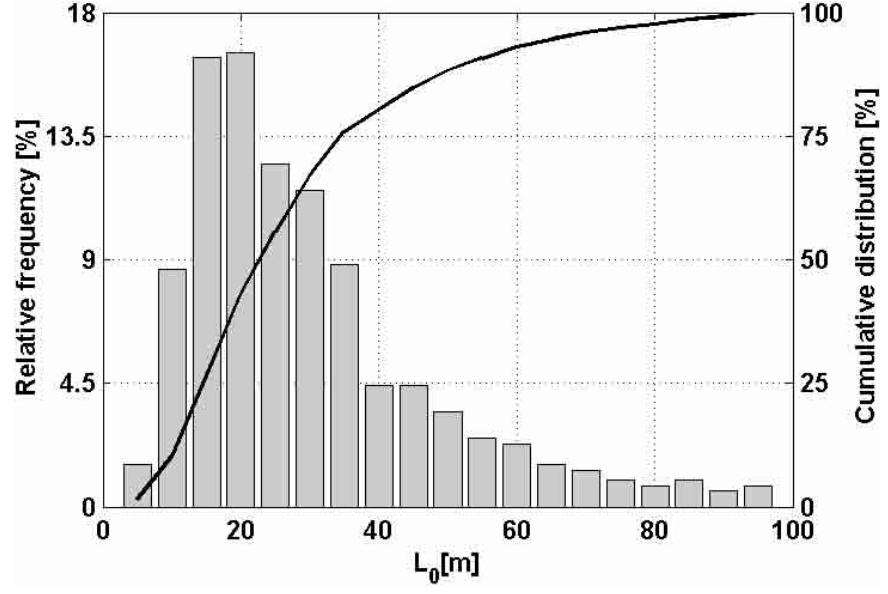

Fig. 4. Histogram of $\mathcal{L}_{0}$ for the whole GSM run.

\subsection{The seeing}

\subsubsection{Comparison of GSM and DIMM values}

Figure 6 shows the seeing measured by the GSM and the $\operatorname{DIMM}\left(\epsilon_{\mathrm{GSM}}\right.$ and $\left.\epsilon_{\mathrm{DIMM}}\right)$ during the night from 2000 December 3 rd to 4 th. A very good agreement can be noticed. However, this was not observed in all the measurements. An example of disagreement is shown in Fig. 7. During that night, the microthermal sensors delivered the optical turbulence strength $\left(C_{n}^{2}\right)$

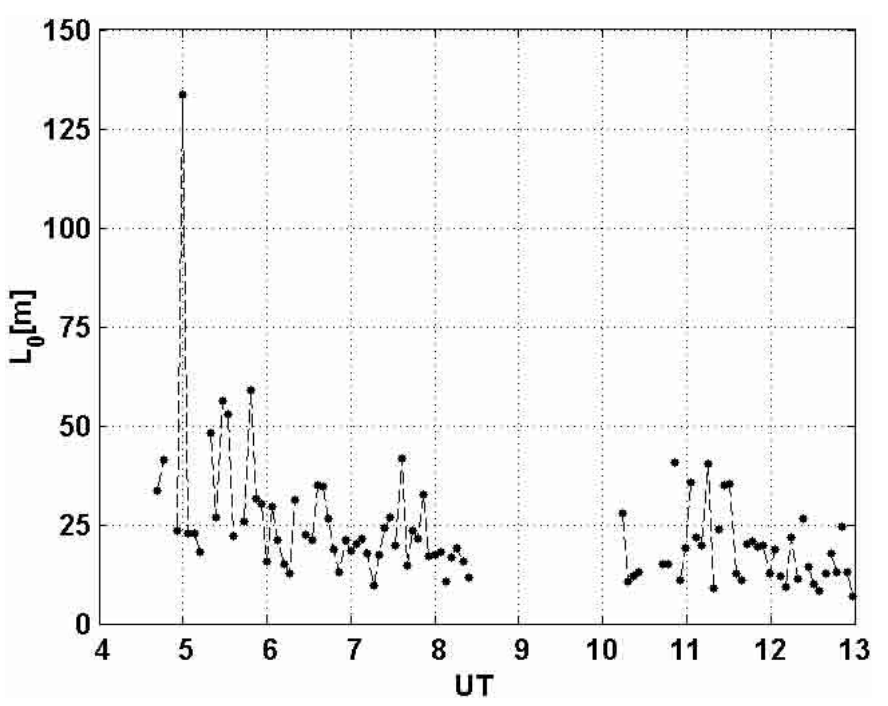

Fig. 5. $\mathcal{L}_{0}$ values measured by the GSM during the night of 2000 December 8th to 9th.

at altitudes of $2.3,3,4,8.3$ and $15 \mathrm{~m}$. Using the $C_{n}^{2}$ values obtained at 2.3,3, 4 and $8.3 \mathrm{~m}$, we calculated $\epsilon_{\text {DIMM } @ 2 \mathrm{~m}}$ which is the seeing that the DIMM would have measured if it had been placed at the same altitude as that of GSM, and is given by:

$\epsilon_{\mathrm{DIMM} @ 2 \mathrm{~m}}^{5 / 3}=\epsilon_{\mathrm{DIMM}}^{5 / 3}+15.86 \lambda^{-1 / 3} \int_{2.3 \mathrm{~m}}^{8.3 \mathrm{~m}} C_{N}^{2}(h) \mathrm{d} h$. 


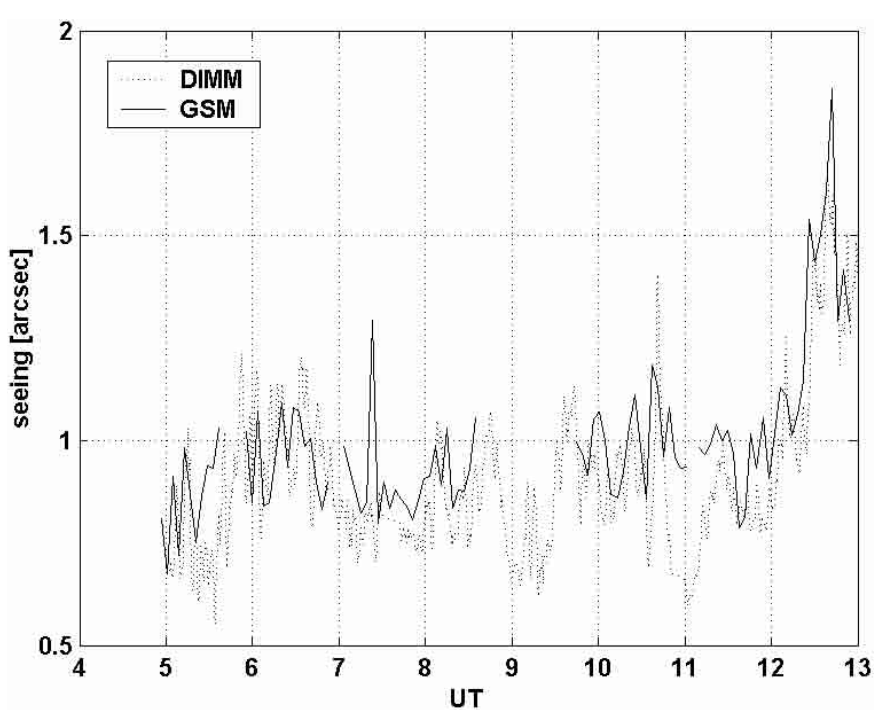

Fig. 6. Seeing measured by the GSM (full line) and the DIMM (dotted line) during the night of 2000 December 3th to 4th. A very good agreement is noticed.

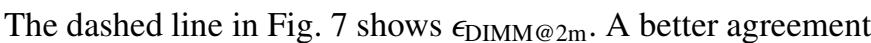
is noticed, but a non-negligible difference is still present mostly during the second half of the night. This difference might be explained by the fact that the GSM delivers seeing values extrapolated to an integration time of $0 \mathrm{~ms}$ (Sect. 2.2), while the DIMM give seeing values obtained with a $10 \mathrm{~ms}$ integration time. Martin (1987) presented the influence of the integration time on the measurement of the variance of the differential as well as non-differential image motion. The theoretical results can be applied directly to compute the underestimation error in the DIMM measurements due to a too-long exposure time. Following the author, to properly correct every seeing value delivered by the DIMM, we would need measurements of the turbulence and wind profiles $\left(C_{n}^{2}(h)\right.$ and $\left.v(h)\right)$ obtained at the same time as the seeing data, which unfortunately we do not have. Nevertheless, we used turbulence and wind profiles measured at the site with the generalized Scidar of the DAUNSA, in May 2000 , to have an estimate of the statistical distribution of the error due to the exposure time. From every pair of simultaneous profiles, $C_{n}^{2}(h)$ and $v(h)$, and using Martin's results, we compute the seeing values that would be obtained by a DIMM with a $10-\mathrm{ms}$ and $0-\mathrm{ms}$ exposure time, $\epsilon_{10 \mathrm{~ms}}$ and $\epsilon_{0 \mathrm{~ms}}$, respectively. The value of $\epsilon_{10 \mathrm{~ms}}$ depends on the direction of the measured image motion and on the wind direction, both with respect to the sub-pupil separation. We decided to consider the worst set of parameters: that in which $\epsilon_{10 \mathrm{~ms}}$ is minimum. The attenuation factor due to the exposure-time is given by $f=\epsilon_{10 \mathrm{~ms}} / \epsilon_{0 \mathrm{~ms}}$. 1021 values of $f$ were computed using $C_{n}^{2}(h)$ and $v(h)$ profiles measured during 8 nights. The median value obtained for $f$ is 0.84 , its standard deviation is 0.09 , and its minimum and maximum values are 0.54 and 0.99 . This range of values of $f$ are consistent with the ratio $\epsilon_{\mathrm{DIMM}} @ 2 \mathrm{~m} / \epsilon_{\mathrm{GSM}}$ which can be deduced from the dashed and full lines in Fig. 7. These results support the hypothesis that the difference in the exposure times employed by the DIMM and the GSM is partly responsible of

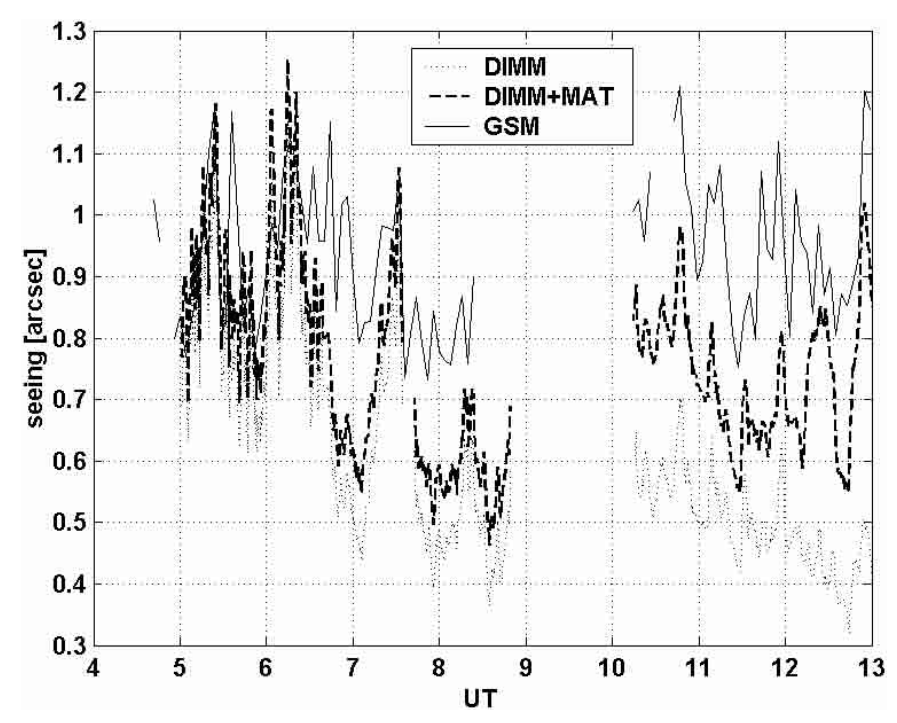

Fig. 7. Same as Fig. 6 but for the night of 2000 December 8th to 9th. The seeing measured by both instruments show a considerable difference after 6 hrs UT. The dashed line represents the seeing that the DIMM would have measured if it had been placed at the same altitude as that of the GSM (see text).

the differences in the seeing measurements obtained by those instruments.

\subsubsection{DIMM results}

The histogram of the seeing values measured with the DIMM during the December 2000 campaign shows a bimodal distribution, with peaks centered at $0.50^{\prime \prime}$ and $0.75^{\prime \prime}$, as shown in Fig. 8. The histogram of the seeing measured with the GSM confirmed the bimodal distribution. Interestingly, the two seeing regimes took place during isolated intervals of several nights, instead of having occurred during different entire nights. This fact is noticed in Fig. 9. Such a behavior shows that the seeing conditions can vary considerably during a single night. From plots of the seeing vs. time for each night we notice that the seeing variation in a single night can be even much larger $\left(>1^{\prime \prime}\right)$ than that shown by the two peaks in the histogram of Fig. 8.

We performed a statistical analysis of all seeing measurements obtained during the five observational campaigns carried out with the DIMM. A total of 18762 measurements where gathered during 31 nights. As explained in Sect. 2.3, in May and October 2000, the pupil of the DIMM was $2 \mathrm{~m}$ above the ground, while in December 2000 and October 2001 its altitude was $8.3 \mathrm{~m}$. In order to analyse the entire DIMM data together as if the instrument would have always been at an $8.3-\mathrm{m}$ altitude, we estimated the seeing values that would have been measured at $8.3 \mathrm{~m}\left(\epsilon_{\mathrm{DIMM}} @ 8 \mathrm{~m}\right)$, by subtracting the mean turbulence contribution from 2 to $8.3 \mathrm{~m}\left(\left\langle\int_{2 \mathrm{~m}}^{8.3 \mathrm{~m}} C_{N}^{2}(h) \mathrm{d} h\right\rangle\right)$ to the DIMM data measured at $2 \mathrm{~m}\left(\epsilon_{\mathrm{DIMM}} @ 2 \mathrm{~m}\right)$. The precise formula is:

$\epsilon_{\mathrm{DIMM} @ 8 \mathrm{~m}}^{5 / 3}=\epsilon_{\mathrm{DIMM} @ 2 \mathrm{~m}}^{5 / 3}-15.86 \lambda^{-1 / 3}\left\langle\int_{2 \mathrm{~m}}^{8.3 \mathrm{~m}} C_{N}^{2}(h) \mathrm{d} h\right\rangle$, 


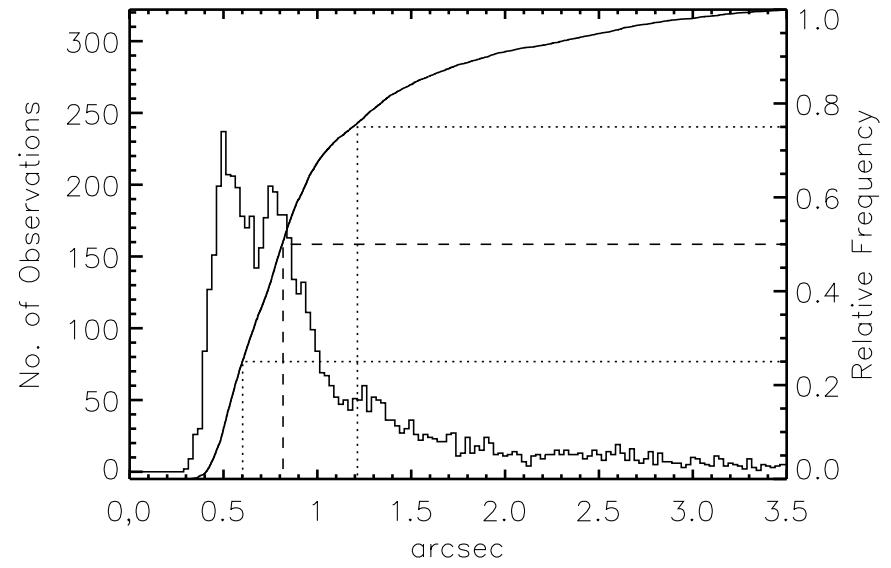

Fig. 8. Histogram and cumulative distribution of the seeing values obtained with the DIMM during the December 2000 observing campaign.

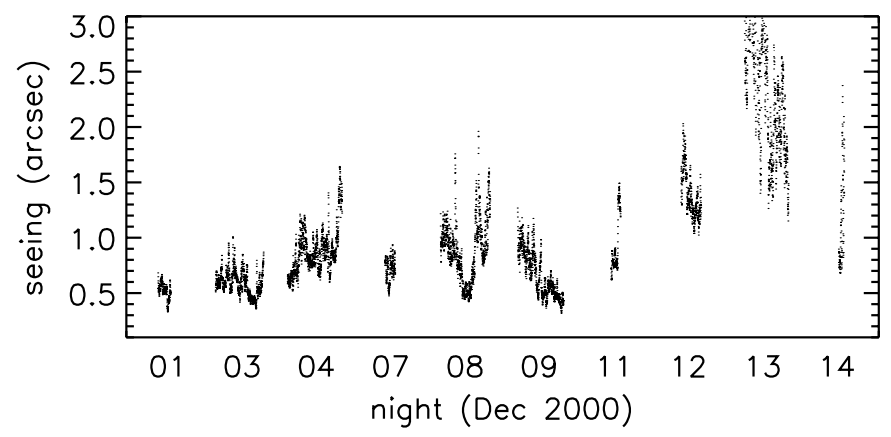

Fig. 9. Seeing measured with the DIMM during the December 2000 observing campaign.

where the average, represented by the signs \langle\rangle , is performed over the measurements obtained with the instrumented mast during the whole May 2000 campaign. The histogram and the cumulative distribution of the resulting entire DIMM data set are shown in Fig. 10. The median value is $0.77^{\prime \prime}$, and the 1 st and 3rd quartiles are $0.61^{\prime \prime}$ and $0.99^{\prime \prime}$. The histogram of the logarithm of the seeing values is well fitted by a Gaussian, which shows that the measured seeing follows a log-normal distribution. The fitted Gaussian is centered at $-0.14 \log (\operatorname{arcsec})$ and has a dispersion of $0.15 \log (\operatorname{arcsec})$.

The seeing values measured by the DIMM are comparable to those found by Avila et al. (1998) using a generalized Scidar at the $2.1 \mathrm{~m}$ telescope, and are somewhat larger than those found by Echevarría et al. (1998) using non-differential seeing monitors.

\subsection{Correlations with wind data}

We investigated the correlation between the wind vector at ground level and two optical turbulence parameters: seeing and outer scale. An ultrasonic anemometer was in operation during the December 2000 observations. It was installed on top of the DIMM tower. The anemometer delivered a measurement of the three-dimensional wind vector every $0.5 \mathrm{~s}$. The wind values corresponding in time with seeing or outer scale values were found by linear interpolation of the wind data set.

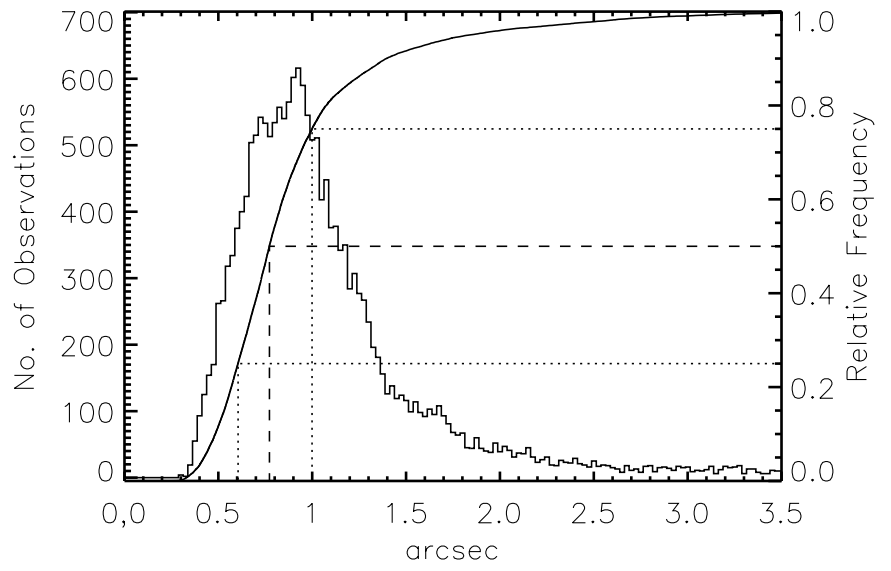

Fig. 10. Histogram and cumulative distribution function of the seeing values obtained with the DIMM during the four campaigns described in Sect 2.3.

Figure 11 shows the wind direction versus the seeing measured by the GSM, for the nights of 2000 December 6-13th, excepting 9th when the GSM gathered no data (see Fig. 3). Low seeing values $\left(\lesssim 2^{\prime \prime}\right)$ are spread over the entire range of the measured wind directions, showing no particular correlation, whereas higher seeing values seem to be associated with wind directions between $200^{\circ}$ and $240^{\circ}$ (i.e. wind coming from the SW). Similarly, in Fig. 12, which shows the wind direction versus $\mathcal{L}_{0}$ for the same nights as in Fig. 11, only high outer scale values ( $\gtrsim 100 \mathrm{~m}$ ) seem to be correlated with a given wind direction, namely between $180^{\circ}$ and $220^{\circ}$ (i.e. wind coming from the SSW). These suggested correlations must not be taken as a definitive result, because there are too few correlated data in both cases. However, interestingly, $\mathcal{L}_{0}$ values larger than $100 \mathrm{~m}$ were not detected during an isolated period of time. They were measured occasionally during the whole observing campaign of 2000 December, which reinforces the correlation found. Note, from Fig. 3, that seeing values higher than $2^{\prime \prime}$ come from the nights of December 10-12th, whereas $\mathcal{L}_{0}$ values larger than $100 \mathrm{~m}$ come mainly from the night of December 7th, however these high values of both parameters seem to be correlated with winds blowing from SSW. This means that the wind direction is not the only parameter that might affect seeing and $\mathcal{L}_{0}$ values.

\subsection{The isoplanatic angle}

The median values of the isoplanatic angle for each night are given in Table 1. The measurements and the histogram are plotted in Fig. 3. The median value is $2.05^{\prime \prime}$ with a standard deviation of $0.90^{\prime \prime}$. This median value is slightly larger than those measured by Avila et al. (1998) in March and April 97 during Generalized Scidar runs. One can notice the unusual large fluctuations of $\theta_{0}$ from $0.5^{\prime \prime}$ to more than $4^{\prime \prime}$ during a same night. This occurred in several nights. These large fluctuations of $\theta_{0}$ might be due to strong fluctuations of the turbulence in a highaltitude layer. In the $C_{n}^{2}$ profiles that we have measured with a Generalized Scidar at the site (Avila et al. 1998, 2001), we have rarely encountered such a situation. 


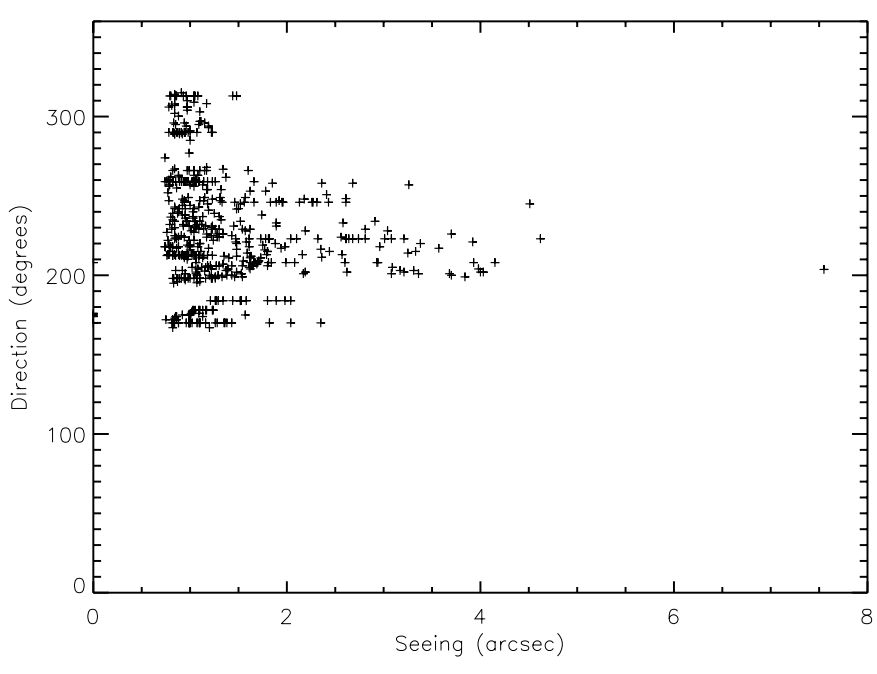

Fig. 11. Wind direction versus the seeing measured with the GSM. The data shown corresponds to the entire December 2000 observing campaign.

\section{Conclusion}

The measurements of $\mathcal{L}_{0}$, with a median value of $27.0 \mathrm{~m}$, confirm the singular uniformity of the statistical distributions of $\mathcal{L}_{0}$ found among all the sites visited by the GSM. We found no evidence of any influence of the trees in the observatory area on $\mathcal{L}_{0}$ values. This is a significant result for the choice of astronomical sites.

Preliminary results suggest that large seeing and $\mathcal{L}_{0}$ values are likely to be found when the wind is blowing from SSW. Further investigations should be performed to confirm this correlation. Unusual large nightly fluctuations of $\theta_{0}$ were detected.

For a few nights, a good agreement was found between the seeing measured by the GSM and by the DIMM. For the rest of the nights, our results suggest that the difference can be explained by the altitude difference of the instruments and by their different integration times. For the first time, the effect of the integration time over seeing measurements performed with a DIMM are estimated using real $C_{n}^{2}$ and velocity profiles. For the sake of comparing the seeing values obtained with exposure times of 0 and $10 \mathrm{~ms}$, using the GSM and the DIMM respectively, it would have been preferable to calculate the attenuation factor due to the 10-ms exposure time $f$ using $C_{n}^{2}$ and velocity profiles measured simultaneously to the seeing values, rather than in a different epoch. However, the set of values of $f$, inferred from the available $C_{n}^{2}$ and $v$ profiles, have sufficient statistical meaning to lead to the conclusion stated above.

The histogram of DIMM values, measured during the campaign of December 2000, shows a peculiar bi-modal distribution centered at $0.5^{\prime \prime}$ and $0.75^{\prime \prime}$. This distribution can be associated to two turbulent regimes able to occur in the same night. The histogram of the DIMM values, measured during all the campaigns from May 2000 to October 2001, show the regular uni-modal log-normal distribution with a median value of $0.77^{\prime \prime}$.

In Sects. 3.3.1 and 3.3.2 we have considered seeing values computed in several ways: with integration times of 0 and $10 \mathrm{~ms}$, and with instruments located at altitudes of 2 and $8.3 \mathrm{~m}$.

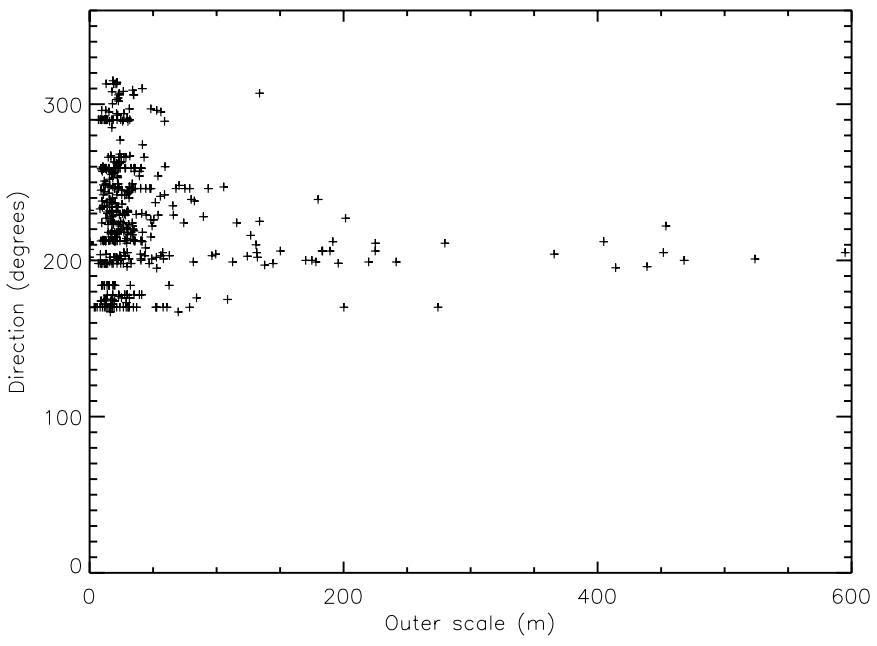

Fig. 12. Wind direction versus the wavefront outer scale. The data shown corresponds to the entire December 2000 observing campaign.

The reader might wonder what are the values that should be retained. It is generally accepted that seeing monitors should be installed at some altitude above the ground, to avoid turbulence on the ground surface like most telescopes do. The altitudes of the seeing monitors vary from 5 to $10 \mathrm{~m}$ approximately. Since 2000 December, the DIMM of the OAN-SPM has been installed on an 8-m-high tower. On the other hand, to avoid filtering effects due to exposure time, the correct procedure for estimating the seeing is to measure it with at least 2 different exposure times and extrapolate the value that would be obtained with a null exposure time. This is the method employed by the GSM, but is very rarely employed by other seeing monitors, like the DIMM of the OAN-SPM, which generally use a 10-ms exposure time. For these reasons, in order to fairly compare seeing values from a variety of sites, we suggest the reader to consider the values reported here obtained with the DIMM at 8-m-hight above the ground and with a 10-ms exposure time: median value of $0.77^{\prime \prime}, 1$ st and 3rd quartiles $0.61^{\prime \prime}$ and $0.99^{\prime \prime}$, keeping in mind that these values should be increased by $16 \%$ to take into account the filtering due to the exposure time (because the median attenuation factor is $f=0.84$ as presented in Sect. 3.3.1), and obtain more exact values of the seeing statistics.

Acknowledgements. A special mention is due to S. Cuevas for his participation in the DIMM measurements of May 2000 and for his continuous support of seeing studies at the IA-UNAM. We are indebted to M. Azouit for lending us the equipment of the micro-thermal sensors and reducing the corresponding data. We also thank the OAN-SPM staff for their help during the observing campaigns. This work was done in the framework of a collaboration between the Instituto de Astronomía of the Universidad Nacional Autónoma de México and the UMR 6525 Astrophysique, Université de Nice-Sophia Antipolis (France). Funding for the site characterization campaign was provided by the grants number J32412E from CONACyT and IN118199 from DGAPA-UNAM, and from the UMR 6525 Astrophysique, Université de Nice-Sophia Antipolis. 


\section{References}

Alvarez, M. 1969, Reporte Técnico No. 5 (Instituto de Astronomía de la Universidad Nacional Autónoma de México), 5

Avila, R., Vernin, J., Azouit, M., et al. 2001, in Site 2000 IAU Technical Workshop on Astronomical Site Evaluation in the Visible and Radio Range, ed. J. Vernin, C. Muñóz-Tuñón, \& Z. Benkhaldoun

Avila, R., Vernin, J., \& Cuevas, S. 1998, Publications of the Astronomical Society of the Pacific, 110, 1106

Borgnino, J. 1990, Appl. Opt., 29, 1863

Echevarría, J., Tapia, M., Costero, R., et al. 1998, Rev. Mex. Astron. Astrofís., 34, 47

Ehgamberdiev, S. A., Baijumanov, A. K., Ilyasov, S. P., et al. 2000, A\&AS, 145, 293

Fried, D. 1982, J. Opt. Soc. Am. A, 72, 52

Hiriart, D., Goldsmith, P. F., Skurtskie, M. F., \& Salas, L. 1997, Rev. Mex. Astron. Astrofís., 33, 59

Martin, F., Tokovinin, A., Agabi, A., Borgnino, J., \& Ziad, A. 1994, A\&AS, 108, 173
Martin, F., Conan, R., Tokovinin, A., et al. 1998a, Report on the GSM Measurement Campaign at the Paranal Observatory, ESO report VLT-TRE-UNI-17440-0006, ESO

Martin, F., Tokovinin, A., Ziad, A., et al. 1998b, Report on G.S.M. Measurement Campaign at La Silla, ESO report VLT-TRE-UNI17440-0005, ESO

Martin, H. 1987, JASP, 99, 1360

Masciadri, E., Avila, R., \& Sánchez, L. J. 2002, A\&A, 382, 378

Michel, R., Bohígas, J., Arroyo, E., \& Zazueta, S. 2001, Rev. Mex. Astron. Astrofís., 37, 165

Roddier, F. 1981, in Progress in Optics, Vol. XIX (E. Wolf)

Sarazin, M., \& Roddier, F. 1990, A\&A, 227, 294

Tapia, M. 1992, Rev. Mex. Astron. Astrofís., 24, 179

Tokovinin, A., Ziad, A., Martin, F., et al. 1998b, in SPIE, vol. 3353, Adaptive Optical System Technologies, ed. D. Bonaccini, \& R. Tyson, 1155

Vernin, J., \& Muñoz-Tuñón, C. 1995, PASP, 107, 265

Ziad, A., Conan, R., Tokovinin, A., Martin, F., \& Borgnino, J. 2000, Appl. Opt., 39, 5415 